\title{
BIPARTITION POLYNOMIALS, THE ISING MODEL AND DOMINATION IN GRAPHS
}

\author{
MARKus DOD ${ }^{1}$ \\ Faculty Mathematics, Sciences, Computer Science \\ University of Applied Sciences Mittweida \\ e-mail: mdod@hs-mittweida.de \\ TOMer KoteK ${ }^{2}$ \\ Institut für Informationssysteme 184/4 \\ Technische Universität Wien, Vienna, Austria \\ e-mail: kotek@forsyte.at \\ JAMEs PREen \\ Mathematics \\ Cape Breton University \\ Sydney, Canada \\ e-mail: james_preen@capebretonu.ca \\ AND \\ Peter Tittmann \\ Faculty Mathematics, Sciences, Computer Science \\ University of Applied Sciences Mittweida \\ e-mail: peter@hs-mittweida.de
}

\begin{abstract}
This paper introduces a trivariate graph polynomial that is a common generalization of the domination polynomial, the Ising polynomial, the matching polynomial, and the cut polynomial of a graph. This new graph polynomial, called the bipartition polynomial, permits a variety of interesting
\end{abstract}

\footnotetext{
${ }^{1}$ Research supported by ESF.

${ }^{2}$ Research supported by the Austrian National Research Network S11403-N23 (RiSE) of the Austrian Science Fund (FWF), and by the Vienna Science and Technology Fund (WWTF) grant PROSEED.
} 
representations, for instance as a sum ranging over all spanning forests. As a consequence, the bipartition polynomial is a powerful tool for proving properties of other graph polynomials and graph invariants. We apply this approach to show that, analogously to the Tutte polynomial, the Ising polynomial introduced by Andrén and Markström in [3], can be represented as a sum over spanning forests.

Keywords: domination, Ising model, graph polynomial.

2010 Mathematics Subject Classification: 05C30, 05C31, 05 C69.

\section{REFERENCES}

[1] W. Ahrens, Mathematische Unterhaltungen und Spiele (B.G. Teubner, Leipzig, Berlin, 1921).

[2] M. Aigner, A Course in Enumeration (Springer, Heidelberg, 2007).

[3] D. Andrén and K. Markström, The bivariate Ising polynomial of a graph, Discrete Appl. Math. 157 (2009) 2515-2524. doi:810.1016/j.dam.2009.02.021

[4] J.L. Arocha, Propiedades del polinomio independiente de un grafo, Ciencias Matemăticas 5 (1984) 103-110.

[5] J.L. Arocha and B. Llano, Meanvalue for the matching and dominating polynomial, Discuss. Math. Graph Theory 20 (2000) 57-69. doi:10.7151/dmgt.1106

[6] A.E. Brouwer, The number of dominating sets of a finite graph is odd, preprint, (2009). http://www.win.tue.nl/ aeb/preprints/domin2.pdf.

[7] K. Dohmen and P. Tittmann, Domination reliability, Electron. J. Combin. 19(1) (2012) \#15.

[8] J.A. Ellis-Monaghan and I. Moffatt, The Tutte-Potts connection in the presence of an external magnetic field, Adv. Appl. Math. 47 (2011) 772-782. doi:10.1016/j.aam.2011.02.004

[9] D. Garijo, A. Goodall and J. Nešetřil, Distinguishing graphs by their left and right homomorphism profiles, European J. Combin. 32 (2011) 1025-1053. doi:10.1016/j.ejc.2011.03.012

[10] C. Godsil and I. Gutman, On the theory of the matching polynomial, J. Graph Theory 5 (1981) 137-144. doi:10.1002/jgt.3190050203

[11] C. Godsil and G. Royle, Algebraic Graph Theory (Springer, 2001). doi:10.1007/978-1-4613-0163-9

[12] I. Gutman and F. Harary, Generalizations of the matching polynomial, Util. Math. 24 (1983) 97-106. 
[13] O.J. Heilmann and E.H. Lieb, Theory of monomer-dimer systems, Commun. Math. Phys. 25 (1972) 190-232.

doi:10.1007/BF01877590

[14] C. Hoede and X.-L. Li, Clique polynomials and independent set polynomials of graphs, Discrete Math. 125 (1994) 219-228. 10.1016/0012-365X(94)90163-5

[15] T. Kotek, Complexity of Ising polynomials, Combin. Probab. Comput. 21 (2012) $743-772$. doi:10.1017/S0963548312000259

[16] T. Kotek, J. Preen and P. Tittmann, Subset-sum representations of domination polynomials, Graphs Combin. 30 (2014) 647-660. doi:10.1007/s00373-013-1286-z

[17] T. Kotek, J. Preen and P. Tittmann: Domination polynomials of graph products, submitted.

[18] K. Markström, The general graph homomorphism polynomial: Its relationship with other graph polynomials and partition functions, arXiv preprint arXiv:1401.6335 (2014).

[19] B. Mohar, Graph laplacians, in: Topics in Algebraic Graph Theory, L.W. Beineke and R.J. Wilson (Eds.), Cambridge University Press (2004) 113-136.

[20] A.D. Scott and G.B. Sorkin, Polynomial constraint satisfaction problems, graph bisection, and the Ising partition function, ACM Transactions on Algorithms (TALG) 5 (2009) 4. doi:10.1145/1597036.1597049

[21] W.T. Tutte, A contribution to the theory of chromatic polynomials, Canadian J. Math. 6 (1954) 80-91. doi:10.4153/CJM-1954-010-9

[22] J.M.M. van Rooij, Polynomial space algorithms for counting dominating sets and the domatic number, in: CIAC 2010, LNCS 6078, T. Calamoneri and J. Diaz (Eds.)(2010) 73-84.

Received 23 January 2014

Revised 16 September 2014

Accepted 16 September 2014 